\title{
PARP INHIBITION IN ATHEROSCLEROSIS $\Lambda$ ND ITS EFFECTS ON Dendritic Cells, T Cells and Auto-Antibody Levels
}

\author{
C. Erbel ${ }^{1}$, J. Achenbach ${ }^{1}$, M. Akhavanpoor ${ }^{1}$, T. J. Dengler ${ }^{1}$, F. Lasitschka ${ }^{2}$, C. A. Gleissner ${ }^{1}$, F. Bea $^{1}$, \\ H. A. Katus ${ }^{1}$, G. Szabo ${ }^{3}$ \\ ${ }^{1}$ Department of Cardiology, ${ }^{2}$ Institute of Pathology, ${ }^{3}$ Department of Cardiac Surgery, \\ Univcrsity of Heidelberg, Germany
}

\begin{abstract}
Objective: Atherosclerosis is a chronic inflammatory process. Poly(ADP-ribose) polymerase-1 (PARP), a nuclear enzyme linked to DNA repair, has been shown to be involved in atherogenesis; however, the effects on dendritic cells, T cells and serum auto-antibody levcls are not fully understood.

Methods: Male Apoe $/$ - mice on a western diet werc treated with the PARP inhibitor INO-1001 ( $\mathrm{n}=15)$, while the control group $(n=15)$ received $5 \%$ glucose solution for 10 weeks.

Results: Inhibition of PARP markedly reduced atherosclerotic lesion development $(\mathrm{p}=0.001)$. Immunohistochemistry and $\mathrm{mRNA}$ analysis revealed a reduced inflammatory compound inside the lesion. Focusing on dendritic cells, INO-1001 reduced number of cells ( $p$ $=0.04)$, grade of activation, represented by $I / 12(\mathrm{p}=$ $0.04)$ and $C d 83(p=0.03)$, and grade of attraction, represented by $M i p 3 \alpha(p=0.02)$ in the plaque. Furthermore, INO-1001 decreased number of T lymphocyte $(p=0.003)$ in the lesion and grade of activation after stimulation with oxLDL in vitro. Moreover, serum IgM antibody levels to oxLDL were significantly lower in INO-1001 treated mice $(\mathrm{p}=0.03)$.

Conclusions: Functional blockade of PARP by INO1001 reduces atherosclerotic lesion development. The anti-atherogenic effect is beside already known mechanisms also moderated due to modulation of DC and T cell invasion and activation, DC attraction as well as IgM antibody levels to oxLDI.
\end{abstract}

Key words: PARP, atherosclerosis, inflammation, oxLDL, dendritic cells, ' $T$ cells

\section{INTRODUCTION}

A chronic (auto)immune response of the arterial wall is a critical mechanism in the development of atherosclerosis $[1,2]$. The disease process is associated with local formation of modified auto-antigens like oxidized low-density lipoprotein (oxT.DL), that are targeted by both the innate and adaptive immune system[2]. Inflammatory cells such as macrophages (MØ), T-lymphocytes and dendritic cells (DC) are believed to bc mainly involved in the initiation and progression of atherogenesis $[2,3]$.

Free radicals react with key organic substrates such as lipids to generate oxLDL. Oxidation of these bio- molecules may impair their biological function and may contribute to initiation or progression of atherosclerosis. Free radicals further interact with isolated or cellular DNA eventually leading to DNA strand breaks and/or base modifications [4]. Cells can respond to DNA strand damage by subsequent activation of the nuclear enzyme poly(ADPribose) polymerase-1 (PARP1) [4]. PARP-1 functions primarily as a DNA damage sensor in the nucleus and mediates the cellular response to DNA strand breaks (sce review Pacher et al. 2008 [5]). Recent studies has suggested that in addition to DNA damage repair, PARP-1 has several other important mode of actions. One of these mechanism is the induction of an energy consuming, futile repair cycle that eventually leads to cellular dysfunction and necrotic cell death (see review Pacher et al. 2008 [5]). PARP-1 in addition modulates inflammation and activation of dendritic cells (DC) thus exerting important effects on inflammation [6]. A previous study further suggested that in vitro PARP inhibition decreases the inflammatory response, reduces oxidative tissue damage, selectively promotes foam cell death, protects endothelial cells (EC) and smooth muscle cells (SMC) from injury by $\mathrm{H}_{2} \mathrm{O}_{2}$, oxidized cholesterol, or tumor necrosis factor [7]. Moreover, PARP-1 inhibition was found to have a beneficial effect of PARP-1 blockade in atherogenesis and possible underlying mechanisms were provided [8-10]. Interestingly, some of the findings of possible mechanisms differ between the groups. In addition, the influence of PARP-1 inhibition on atherogenic cell types (DC, T cells, endothelial cells (EC)) and possible autoimmune responses (oxI.DL dependent activation of cells and serum autoantibody levels against oxLDL) in the lesion are not previously investigated or not fully understood. To investigate how PARP accelerates atherogenesis, apolipoprotein $E$ knockout mice $\left(\right.$ Apoe $\left.^{-/}\right)$were treated with the PARP inhibitor INO-1001.

\section{MATERIAL AND METHODS}

\section{ANIMALS}

Male Apoe $/$ - mice 8 weeks of age (strain B6.129P2) were kept within the animal care facility of the University of Heidelberg. Mice were fed a Western-type diet (Altromin, Germany) composed of $21 \%$ fat by weight $(0.15 \%$ cholesterol and $19.5 \%$ casein without sodium cholate). The therapy group received $1 \mathrm{mg} / \mathrm{kg}$ body 
weight/day of PARP blocking agent INO-1001 intraperitoneally, dissolved in 5\% glucose (Inotek Pharmaceuticals, Beverly, $\mathrm{MA})(\mathrm{n}=15)$, while the control group received $5 \%$ glucose solution $(\mathrm{n}=15)$ for 10 weeks. The housing and care of animals and all other study procedures done in the study were in accordance with the guidelines and regulations of the local Animal Care Committee (IRB approval info: AZ 359185.81/G-41/08).

\section{Tissue Processing}

For RNA isolation, the aortic arch was dissected and snap-frozen as described previously [11]. The entire aortic root, a predilection site for lesion development in Apoe ${ }^{-/}$mice [12], was serially sectioned $(5 \mu \mathrm{m})$, and stained with Oil Red $\mathrm{O}$ as described previously [11]. Beginning with the first slide containing all three valves, a section was stained every $75 \mu \mathrm{m}$ (about 5-8 sections/staining/aortic root).

\section{Determination of Blood Cell Composition as WELl as PLASMa LiPID CONCENTRATION AND DETERMINATION OF ANTIBODY TITER AND SPECIFICITY}

Total serum cholesterol, high-density lipoprotein(HDL-) and low-density lipoprotein- (LDL-) cholesterol, trigylcerides as well as blood cell count were analyzed by standard methods at the department of clinical chemistry of the chemistry of Heidelberg. ELISA was used to quantify $\operatorname{IgG}$ and $\operatorname{IgM}$ antibody levels against oxLDL (kindly provided by R. Klingenberg in the laboratory of G. Hansson, Karolinska Hospital, Stockholm, Sweden [13]).

\section{IMMUNOHISTOCHEMISTRY}

Aortic root cryosections were fixed in acetone, air dried and incubated with monoclonal antibodies as described previously $[3,11]$. Primary antibodies (CD3, vascular cell adhesion molecule-1 (VCAM-1) (both $\mathrm{BD}$ Pharmingen, USA), CD11c (eBioscience, USA), $\alpha$-smooth muscle actin ( $\alpha$-SM actin)(Dianova, Germany) and MAC-2 (Accurate Chemie) were titrated to optimum performance followed by detection with ABC alkaline phosphatase kit or ABC-IP kit (both Vector Laboratories, Burlingame, CA). MOVAT pentachrome staining was used for plaque composition analysis. The detection of apoptosis was performed using an In Situ Cell Death Detection Kit (Roche, Mannheim, Germany) according to the manufacturer's protocol. A thresholding technique using computerized ImagePro analysis on the aortic root sections was implemented.

\section{Real-time Polymerase Chain Reaction}

To isolate total cellular RNA the RNeasy kit (Qiagen, Hilden, Germany) and the Boehringer cDNA kit (Roche Diagnostics, Mannheim, Germany) for reverse transcription were used according to the manufacturer's instructions. For quantitative PCR (qPCR), Roche real-time PCR kit with SYBR Green (Roche Diagnos- tics, Mannheim, Germany) was used as described previously [11]. The primer sequences are shown in Table 1. RT-PCR conditions were: 45 cycles total, each with $95^{\circ} \mathrm{C}$ for 5 seconds, depending on the primer sequences between 55 and $60^{\circ} \mathrm{C}$ for 10 seconds and $72^{\circ} \mathrm{C}$ for 12 seconds. Data were analyzed on the basis of the relative expression method with the formula relative expression $2^{-\Delta C^{\prime} \Gamma}$, where $\Delta C_{T}$ is the difference in threshold cycle between the gene of interest and the housekeeping gene ( $\beta$-Actin) as a control.

Table 1. Primer sequences (murine (m), forward (FP) and reverse (RP) primer) are shown.

\begin{tabular}{|c|c|}
\hline Name & Sequence \\
\hline mCaspase3 FP & 5'-TGGTGATGAAGGGGTCATT'TATG-3' \\
\hline mCaspase3 RP & 5'-TTCGGCTTTCCAGTCAGACTC-3' \\
\hline $\mathrm{mCD} 3 \varepsilon \mathrm{FP}$ & 5'-A'TGCGGTGGAACACTTTCTGG-3' \\
\hline $\mathrm{mCD} 3 \varepsilon \mathrm{RP}$ & 5'-GCACGTCAACTCTACACTGGT-3' \\
\hline $\mathrm{mMIP}-3 \alpha \mathrm{FP}$ & 5'-GCCTCTCGTACATACAGACGC-3' \\
\hline mMIP-3 $\alpha$ RP & 5'-CCAGTTCTGCTTTGGATCAGC-3' \\
\hline $\mathrm{mCD} 83 \mathrm{FP}$ & 5'-CGCAGCTCTCCTA'TGCAGTG-3' \\
\hline $\mathrm{mCD} 83 \mathrm{RP}$ & 5'-GTGTTTTGGATCGTCAGGGAATA-3' \\
\hline mIL-12 FP & 5'-TGGTTTGCCATCGTTTTGCTG-3' \\
\hline mIL-12 RP & 5'-ACAGGTGAGGTTCACTGTTT'TCT-3' \\
\hline mIFN- $\gamma$ FP & 5'-ATGAACGCTACACACTGCATC-3' \\
\hline $\mathrm{mIFN}-\gamma \mathrm{RP}$ & 5'-CCATCCT'T'TTGCCAGTTCCTC-3' \\
\hline
\end{tabular}

\section{Cell Isolation/Culture/In Vitro EXPERIMENTS}

Murine spleens were removed from anesthetized mice and dispersed through a mesh to generate a single-cell suspension. Isolated cells were washed, following removal of erythrocytes. Cells were resuspended at a concentration of $10^{6}$ cells $/ \mathrm{ml}$ in RPMI medium, supplemented with $10 \%$ FCS, penicillin G (100units/ml) and streptomycin $(100 \mu \mathrm{g} / \mathrm{ml})$. Cells were cultured at $37^{\circ} \mathrm{C}$ in a humidified air containing $5 \% \mathrm{CO}_{2}$. Isolated cells were incubated with $20 \mu \mathrm{g} / \mathrm{ml}$ oxidized LDL ((oxIDL), Sanbio, Beutelsbach) (+/- 100ng/ml INO$1001)^{2}$ for $8 \mathrm{~h}$ and $16 \mathrm{~h}$. Unstimulated cells served as controls.

\section{STATISTICAL ANALYSIS}

All data are presented as median $\pm 75^{\text {th }}$ and $25^{\text {th }}$ percentile. Non-parametric Mann-Whitney U test was used to compare individual groups of animals. A level of $\mathrm{p}<0.05$ was considered statistically significant.

\section{RESULTS}

\section{Animal.BloOd Analysis}

Dosing to achieve therapeutic INO-1001 drug levels was established previously [14]. Based on these results, mice were treated with $1 \mathrm{mg} / \mathrm{kg}$ body weight of the PARP inhibitor INO-1001 $(\mathrm{n}=15)$, dissolved in 5\% 
Table 2. Body weight and height, lipid profile and hematological parameters were measured on the day of tissue harvesting (18 weeks) of INO-1001 treated mice and controls. All values are shown as mean \pm S.D.. n.s. $=$ not significant.

\begin{tabular}{llll}
\hline Parameter & $\begin{array}{l}\text { INO-1001-treated } \\
(\mathrm{n}=15) \pm \text { S.D. }\end{array}$ & $\begin{array}{l}\text { Control } \\
(\mathrm{n}=15) \pm \text { S.D. }\end{array}$ & P value \\
\hline Leukocytes $[/ \mathrm{nl}]$ & $1.80 \pm 0.96$ & $1.73 \pm 0.98$ & $\mathrm{n} . \mathrm{s}$. \\
Erythrocytes $[/ \mathrm{pl}]$ & $7.76 \pm 1.27$ & $7.25 \pm 1.68$ & $\mathrm{n} . \mathrm{s}$. \\
Hemoglobin $[\mathrm{g} / \mathrm{dl}]$ & $12.16 \pm 0.94$ & $11.75 \pm 2.41$ & $\mathrm{n} . \mathrm{s}$. \\
Thrombocytes $[/ \mathrm{nl}]$ & $797.38 \pm 308.17$ & $587.33 \pm 504.32$ & $\mathrm{n} . \mathrm{s}$. \\
Triglycerides $[\mathrm{mg} / \mathrm{dl}]$ & $66.43 \pm 21.00$ & $62.75 \pm 32.13$ & n.s. \\
Total Cholesterol $[\mathrm{mg} / \mathrm{dl}]$ & $839.82 \pm 160.22$ & $834.00 \pm 155.09$ & n.s. \\
LDL Cholesterol $[\mathrm{mg} / \mathrm{dl}]$ & $812.68 \pm 158.38$ & $806.00 \pm 152.00$ & n.s. \\
HDL Cholesterol $[\mathrm{mg} / \mathrm{dl}]$ & $13.93 \pm 5.08$ & $17.10 \pm 10.49$ & n.s. \\
Body weight $[\mathrm{g}]$ & $30.60 \pm 3.10$ & $31.27 \pm 2.80$ & n.s. \\
Height $[\mathrm{cm}]$ & $10.10 \pm 1.32$ & $10.76 \pm 1.63$ & n.s. \\
\hline
\end{tabular}
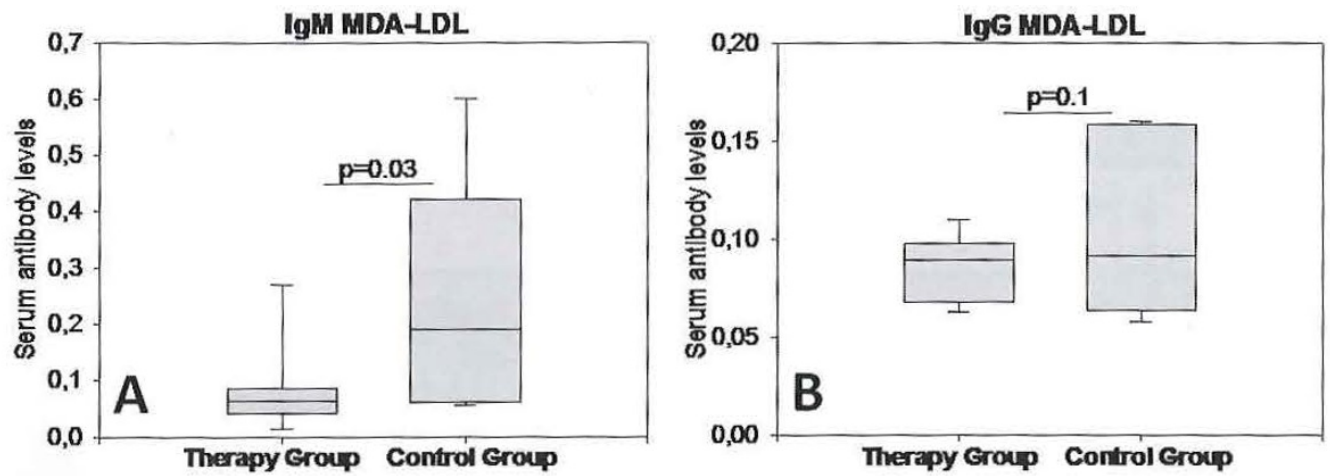

Fig. 1. Antibody serum levels to oxLDL. On the day of tissue harvesting (18 weeks) $\operatorname{IgM}(\mathrm{A})$ and $\operatorname{IgG}$ (B) antibody serum levels to oxLDL were determined by ELISA $(\mathrm{n}=30)$. Results are shown as box plots displaying medians and $25^{\text {th }}$ and $75^{\text {th }}$ percentiles as boxes and 10 th and 90 th percentiles as whiskers. n.s. - not significant.

glucose, while the control group received 5\% glucose solution $(\mathrm{n}=15)$ by intraperitoneal injection daily for 10 weeks. No animal died during the experimental period. Whole blood counts and lipid levels as well as body weight and height are shown in Table 2. Serum IgM antibody levels to oxLDL showed a significant reduction in INO-1001 treated mice compared to controls ( $p=0.03$, Figure (Fig.)1-A). Serum IgG antibody levels to oxLDL were not different between both groups ( $p=0.1$, Fig.1-B).

\section{EFFECTS OF INO-1001 TREATMENT ON Atherosclerotic Plaques in Apoe $/$ - Mice}

Functional blockade of PARP by INO-1001 resulted in significantly reduced atherosclerotic lesions (Fig. 2). The aortic root of INO-1001 treated mice showed reduced fractional stenosis compared to control mice ($32 \% ; 26.1 \% \pm 7 \%$ vs. $38.2 \% \pm 8 \%$; $=0.001$; Fig. 2$)$. Maximum stenosis was markedly reduced accordingly in INO-1001 treated vs. control mice $(-32 \% ; 30.8 \% \pm$ $6 \%$ vs. $45.1 \% \pm 8 \% ; p=0.01$, Fig. 2 ).

To investigate the effect of PARP inhibition on plaque stability, MOVAT pentachrome staining was performed to evaluate collagen content as well as $\alpha$ $\mathrm{SM}$ actin staining to investigate the amount of VSMCs in the lesion. No difference of collagen content (Fig. 3-A-B) and the number of VSMCs was seen between both groups (not shown).

\section{Cellular Infiltration/Activation of Plaque}

Immunohistostaining for CD3 showed reduced numbers of infiltrating $\mathrm{T}$ cells in INO-1001 treated mice compared to controls ( $p=0.02$, Fig. 3-C-F). In addition, $\mathrm{T}$ cell "density (cell number/plaque area $\left(\mathrm{mm}^{2}\right)$ ) was also significantly reduced $(1.39 \pm 0.64$ vs. $4.66 \pm$ $3.40 ; p=0.003$, Fig. 4-A+B). qPCR results corroborated the immunhistology findings: Tissue levels of cDNA transcripts for $C d 3 \varepsilon$, which is a subfragment of the CD3 receptor exclusively expressed by T cells, were distinctly lower in INO-1001 treated than in control mice $(p<0.05$, Fig. 5-A).

Staining for CD11c, a specific marker for DCs, showed no difference in atherosclerotic lesions between both groups, but resulted in significantly reduced numbers in adventitial DC, located adjacent to atherosclerotic lesions, in INO-1001 treated mice (Fig. 4-C, Fig. 6).

Due to reduced DC number, we investigated the levels of the potent chemoattractant for DCs macrophage inflammatory protein- $3 \alpha$ (MIP-3 $\alpha)$ in atherosclerotic lesions. We found that INO-1001 treated compared to control mice showed reduced levels of 

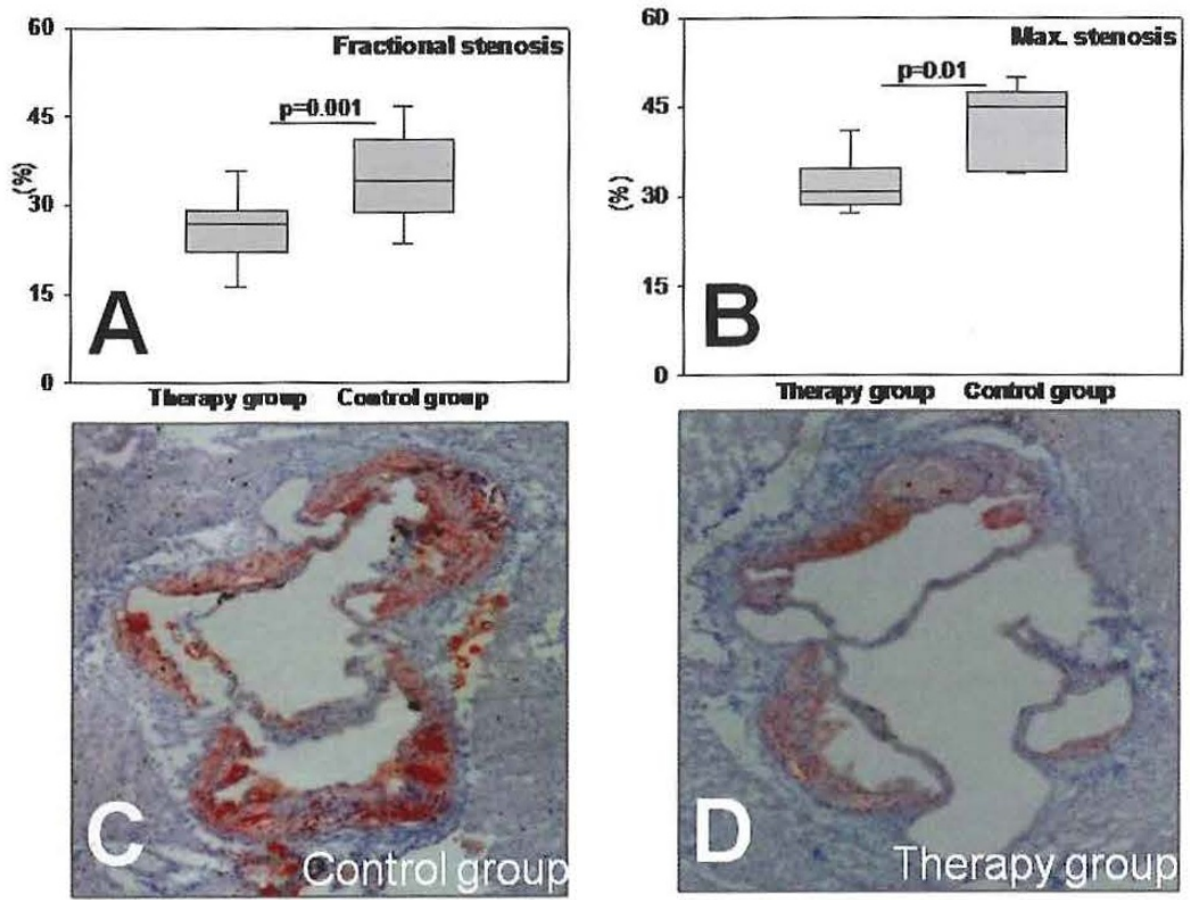

Fig. 2. Effects of PARP-1 inhibition on atherosclerotic plaques in Apoe $\%$ mice. Morphometric quantification of fractional stenosis [\%] (A) and maximum stenosis [\%] (B) of early atherosclerotic lesions were compared between INO-1001 treated $(\mathrm{n}=15)$ and control mice $(\mathrm{n}=15)$. Results are shown as box plots displaying medians and 25th and 75th percentiles as boxes and 10th and 90th percentiles as whiskers. Representative Oil Red O immunohistostainings from aortic root of control (C) and INO-1001 treated (D) mice are shown.

\section{Control}
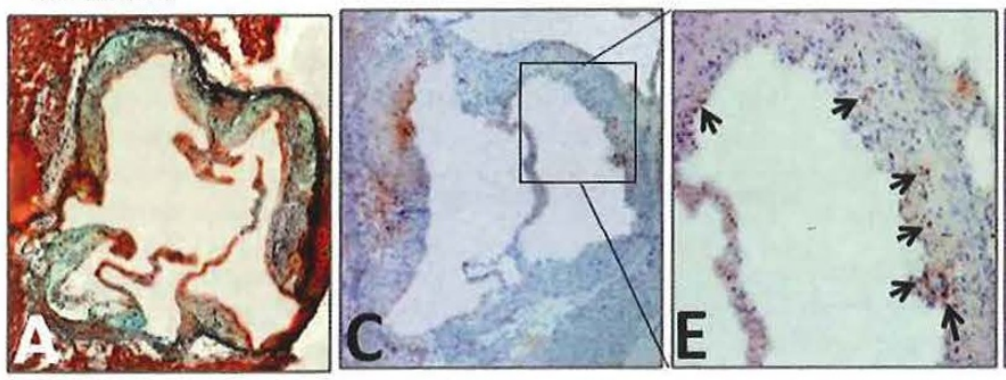

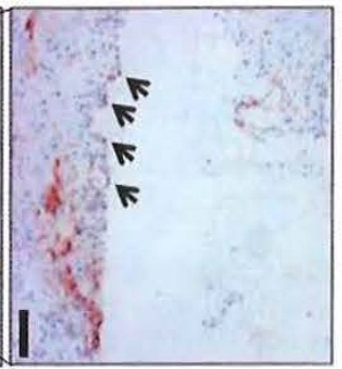

\section{Therapy}
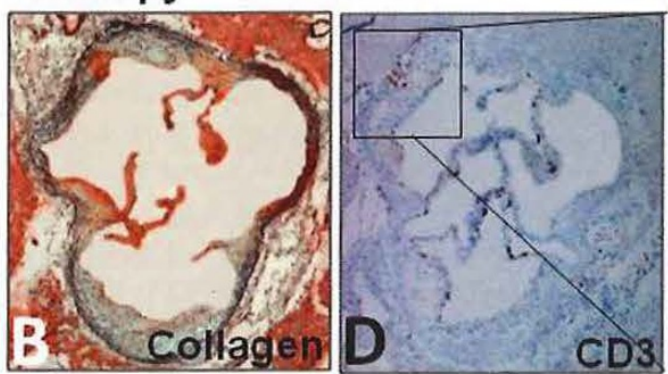
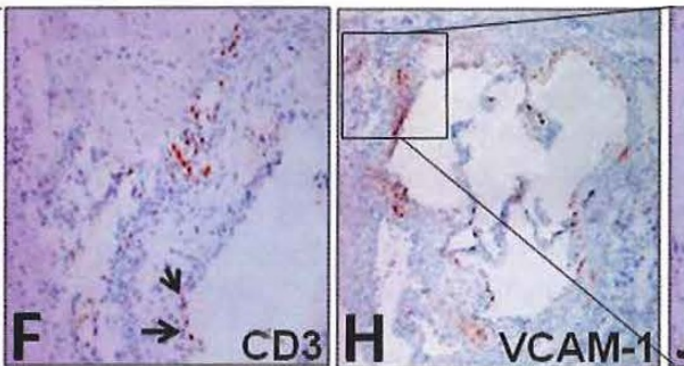

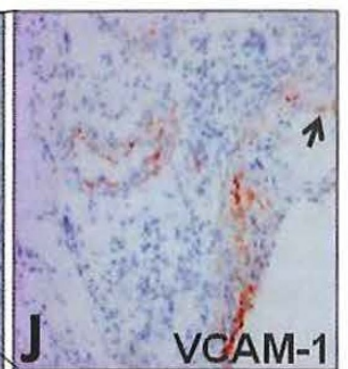

Fig. 3. Cellular composition in atherosclerotic lesions of Apoe $/-$ mice. Representative photomicrographs of immunohistochemistry stainings of collagen (yellow, MOVATs (A+B)), T cells (CD3 (C-F)) as well as activation of the tissue (VCAM-1 (G-J)) and of activated endothelial cells (VCAM-1 $(\mathrm{I}+\mathrm{J}))$ in atherosclerotic lesions $(\mathrm{n}=30), \Lambda-\mathrm{D}$ and $\mathrm{G}-\mathrm{H}$ show $4 \mathrm{x}$ and $\mathrm{E}-\mathrm{F}$ and $\mathrm{I}-\mathrm{J} 20 \mathrm{x}$ magnification.

MIP- $3 \alpha$ in the lesion ( $\mathrm{p}=0.02$, Fig. $5-\mathrm{B}$ ). Focusing on the grade of activation of $\mathrm{DC}$, we measured lesional Cd83 cDNA transcript levels, a specific marker for mature DC, and $1 / 12$, a pro-inflammatory cytokine mainly expressed by DC. Tissue levels of $C d 83$ and $I / 12$ were significantly lower in INO-1001 treated mice compared to controls $(p=0.03$ and $p=0.04$, Fig. 5-C+D).

Immunohistochemistry staining revealed a reduction of the amount of VCAM-1+ cells, a marker for the grade of cellular activation, in atherosclerotic le- sions of INO-1001 treated compared to control mice ( $p=0.002$, Fig. 3-G-J, Fig. 4-D). In addition, the number of VCAM-1 ${ }^{+}$ECs per entire number of ECs was significantly reduced in INO-1001 treated mice compared to controls $(p=0.03$, Fig. 3-G-J). The levels of endothelial nitric oxide synthase (eNos) were significantly reduced in INO-1001 treated compared to control mice ( $p=0.02$, not shown), whereas no difference was seen for the cDNA transcript levels of inducible nitric oxide synthase (iNos) (not shown). 

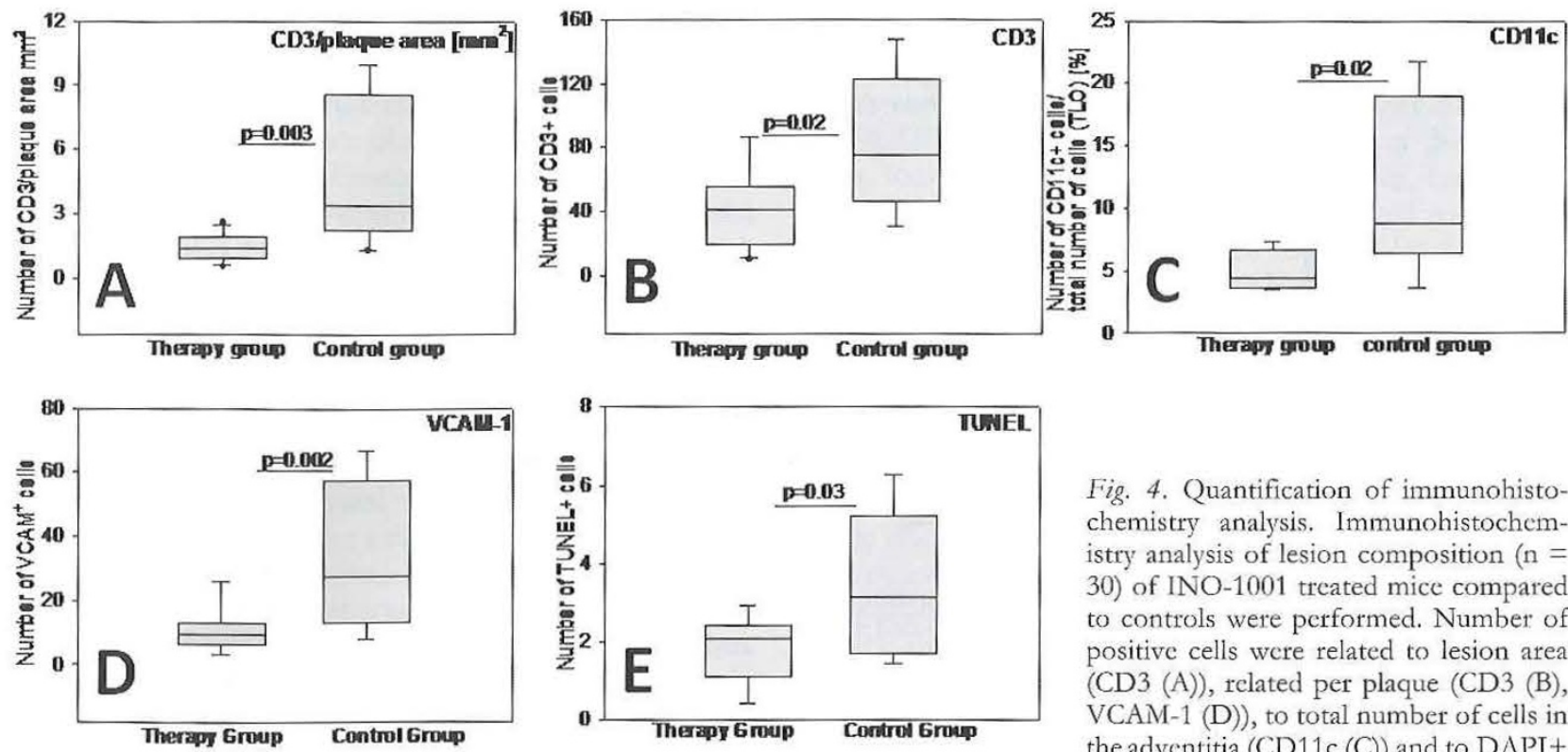

Fig. 4. Quantification of immunohistochemistry analysis. Immunohistochemistry analysis of lesion composition $(\mathrm{n}=$ 30) of INO-1001 treated mice compared to controls were performed. Number of positive cells were related to lesion area (CD3 (A)), related per plaque (CD3 (B), VCAM-1 (D)), to total number of cells in the adventitia (CD11c (C)) and to DAPI+

cells in the lesion (TUNEL (E)) in INO-1001 treated and control mice. Results are shown as box plots displaying medians and 25th and 75 th percentiles as boxes and 10th and 90th percentiles as whiskers. n.s. = not significant.
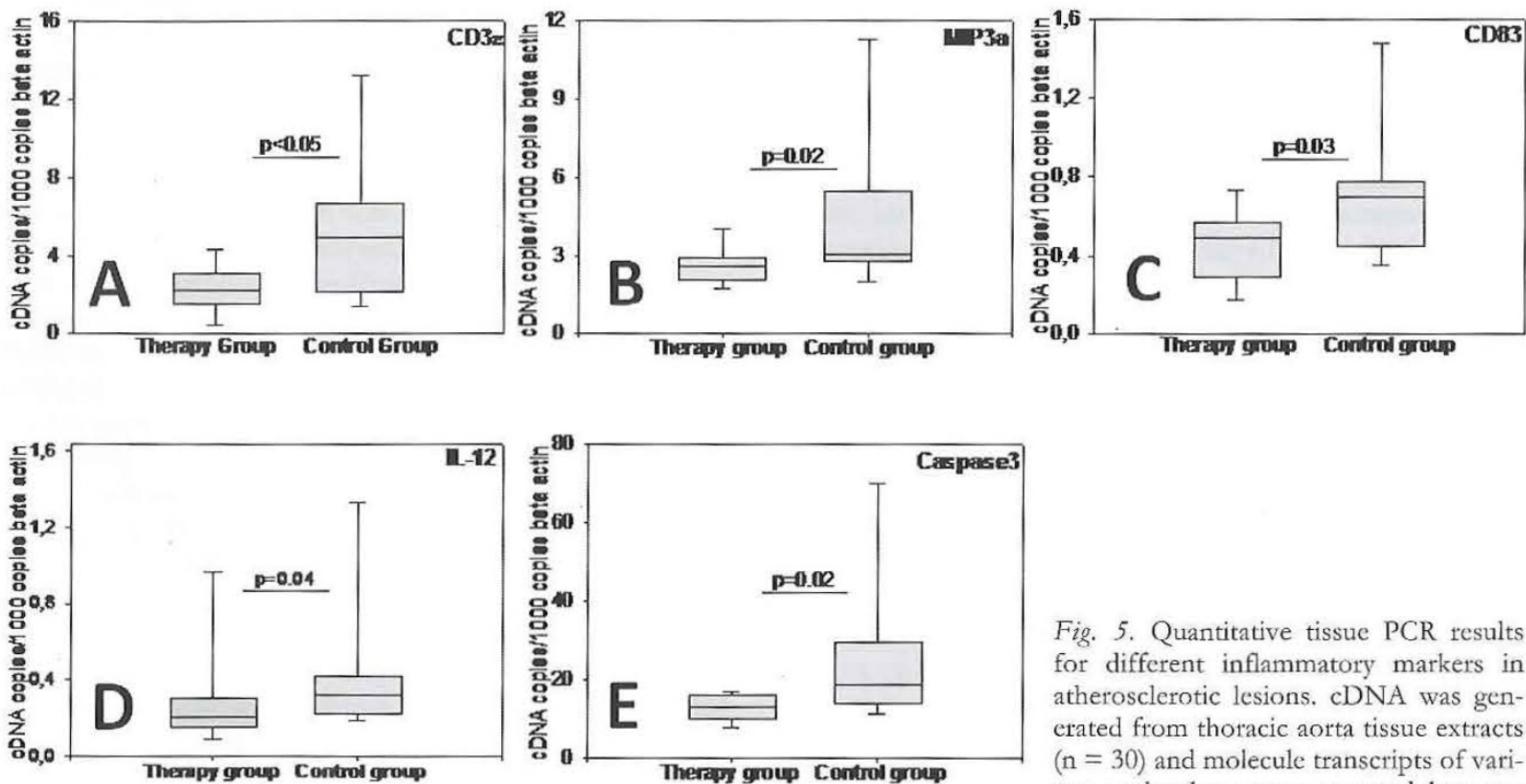

Fig. 5. Quantitative tissue PCR results for different inflammatory markers in atherosclerotic lesions. cDNA was generated from thoracic aorta tissue extracts $(\mathrm{n}=30)$ and molecule transcripts of various molecules were compared between

INO-1001 treated und control animals. Copy numbers were adjusted for copies of the housekeeping gene $\beta$-actin. Results are shown as box plots displaying medians and 25 th and 75 th percentiles as boxes and 10 th and 90 th percentiles as whiskers.
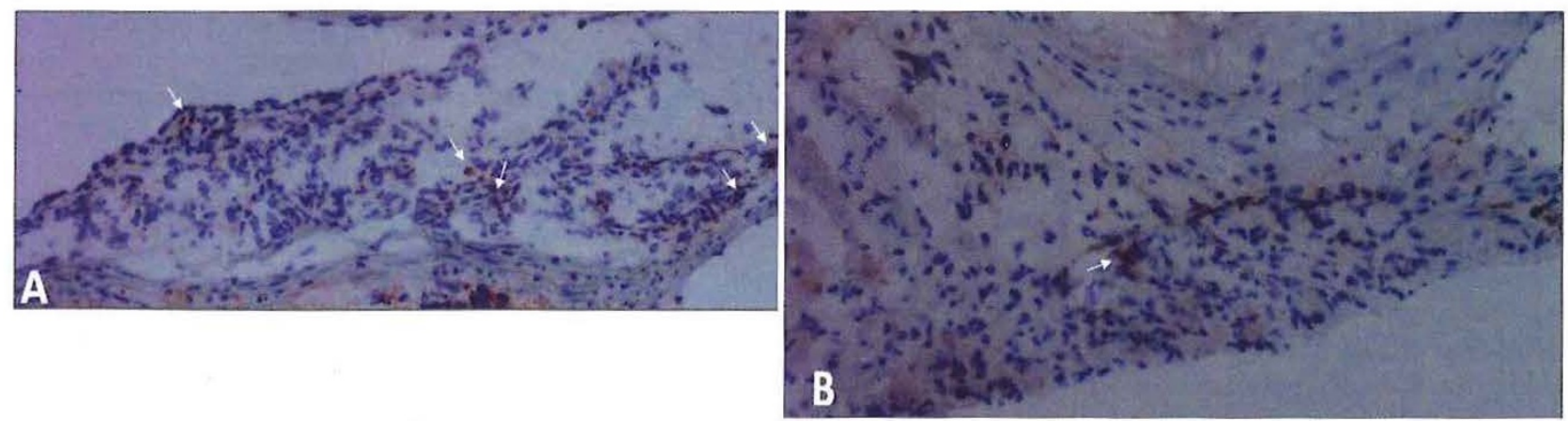

Fig. 6. DCs in the adventitia of Apoe $/-$ mice. Representative photomicrographs of immunohistochemistry staining of adventitial DCs (CD11c) in controls (A) and INO-1001 treated mice (B); 10x magnification. 


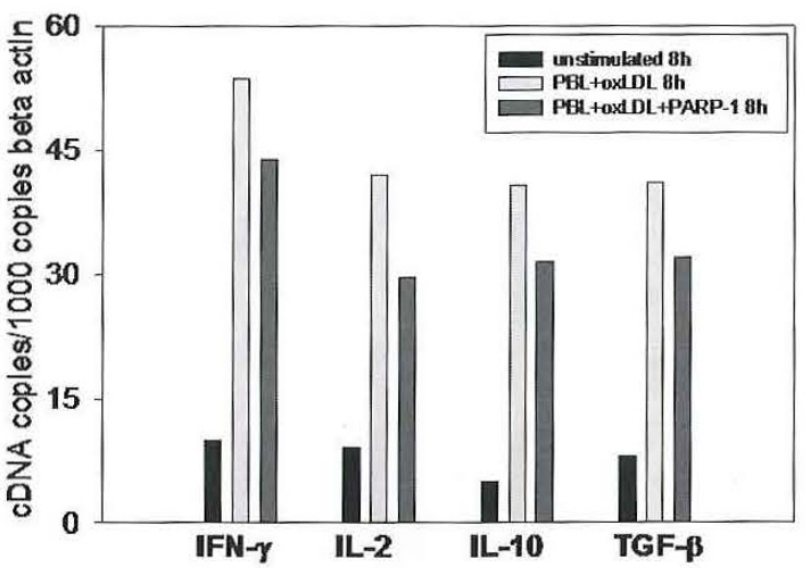

Fig. 7. In vitro effects of PARP-1 inhibition on peripheral blood lymphocytes. Isolated spleen cells were incubated with $20 \mu \mathrm{g} / \mathrm{ml}$ oxLDL. The effects of addition of specific PARP-1 inhibitor INO-1001 on the expression of activation-associated cytokines were measured by qPCR after $8 \mathrm{~h}$. cDNA was generated of incubated cells and molecule transcripts of various molecules were compared between INO-1001 treated und control animals. Copy numbers were adjusted for copies of the housekeeping gene $\beta$-actin. Representative data from one of three independent experiments with similar results.

In order to investigate the amount of $M \varnothing$ in the lesion we immunolabeled frozen sections with MAC-2. MAC-2 staining showed a tendency to reduced numbers of $\mathrm{M} \varnothing$ in INO-1001 treated compared to control mice ( $\mathrm{p}=0.09$; not shown).

To investigate apoptotic cell death in the lesion, TUNEL/DAPI staining on frozen sections was performed. While TUNEL+ stained cells were observed in both groups, blocking PARP-1 led to reduced apoptosis in atherosclerotic plaques ( $p=0.03$, Fig. 4-E), which is in line with lower tissue expression levels of caspase 3 ( $p=0.02$, Fig. 5-E).

\section{INFLUENCE OF INO-1001 ON OXLDL DEPENDENT LYMPHOCYTE ACTIVATION}

In vitro, PARP inhibitor INO-1001 partially reduced the stimulatory capacity of oxLDL on isolated spleen cells after 8h (Fig. 7) and 16h (not shown). Cells incubated with oxLDL in addition to INO-1001 showed reduced production of several cytokines involved in cellular activation and $\mathrm{T}$ cell proliferation $(I l 2, I f n \gamma)$ as well as cytokines $I l 10$ and Tgf $\beta$ compared to peripheral blood lymphocytes incubated with oxLDL without INO-1001 (Fig. 7). Notably, there was no direct apoptotic/toxic effect.

\section{DISCUSSION}

Atherosclerosis is a chronic (auto)immune disease of the arterial wall in which both the innate and adaptive immune systems are involved. Immune mechanisms modulate the development, progression and vulnerability of atherosclerotic lesions.

Our study demonstrates that functional blockade of PARP with the inhibitor INO-1001 leads to a reduction in early atherosclerotic lesion development by
$>30 \%$, but not to increased plaque stability in $\mathrm{Apoe}^{-/-}$ mice fed a high fed diet. INO-1001 treatment resulted in reduced inflammatory cellular infiltration of $\mathrm{T}$ cells and antigen presenting cells such as dendritic cells as well as less tissue activation, in particular EC, represented by VCAM-1, and apoptosis. In addition, dendritic cell activation, represented by $I l 12$ ( $\mathrm{p}=0.04$ ) and $C d 83$ ( $\mathrm{p}=0.03)$, and attraction, represented by MIP $3 \alpha(\mathrm{p}=0.02)$, was reduced. The effects of PARP inhibition on cellular activation was supported by the finding of a partial inhibition of oxJDL-dependent activation of isolated spleen lymphocytes by INO1001 in vitro. Moreover, IgM antibody levels to oxLDL were significantly lower in INO-1001 treated mice compared to controls.

In the aortic root, Apoe $/$ mice on western diet show most rapid development of lesions between 15 and 30 weeks of age [15]. INO-1001 treated mice displayed a significant deceleration of atherosclerotic disease progression during this interval with a significant reduction in the extent of atherosclerotic lesions at 18 weeks of age, i.e. at the end of the 10 week observation period. The extent of protection achieved by the PARP-1 inhibitor INO-1001 in the aortic root was lower compared to PARP-1//-/Apoe ${ }^{-/}(\sim 50 \%)$ or Apoe $^{-/}$mice on high fat diet treated with another PARP inhibitor $(40-50 \%)$ [8-10]. The difference may be due to the experimental setting, the choice of the PARP inhibitor, the different concentrations and half life of the drugs used in the studies, the duration and time period of treatment as well as the type of diet.

Boulares et al. first described an atheroprotective effect of PARP inhibition, which was already confirmed by other groups $[9,10]$. Boulares et al. as well as other groups pointed out several mechanisms [7-10, 16]. The reduced lesion progression seem to be among others based on a downregulated oxidative stress, protective effects on vascular and autonom functions, protection of cells against dysfunction of cell death and on reduced inflammatory cells and molecules such as TNF and monocyte chemotactic protein-1 (MCP-1) for monocytes/macrophage recruitment to the site of inflammation $[7-10,16,17]$. But the reduced levels of MCP-1 did not result in a consistent reduction of the number of macrophages in the lesion between all published studies. While a one study found a significant reduction of the amount of macrophages in atherosclerotic lesions (Apoe $/$-PARP-1/ or the PARP inhibitor PJ34 (N-(6-oxo-5,6-dihydro-phenanthridin-2-yl)-N,Ndimethylacetamide), another study revealed no difference between PARP-1 inhibition and controls in mice on a high fat diet (treatment with thieno[2,3-c]isoquinolin5 -one) $[10,16]$. Based on reduced expression levels of MCP-1, but differential findings of the potency of PARP inhibition on the M $\varnothing$ content in the lesion we investigated the presence of $\mathrm{M} \varnothing$ in the lesion. We also found only a trend observed towards reduced numbers of $\mathrm{M} \varnothing$ in atherosclerotic lesions of INO-1001 treated mice fed a high fat diet. Thus, it can be suggested that the possible reduced macrophage recruitment due to PARP inhibition may not be a crucial mechanism in atherosclerosis, but it needs to be elaborated if the type and the dosage of PARP inhibitor is accountable for the diverse results. 
Beside lesion progression several studies investigate the effect of PARP inhibition of plaque stability, but the findings are not in line with each other [7-9]. Some groups found a positive effect of PARP inhibition on plaque stability $[7,10]$, whereas Chen et al. could not elaborate that [9]. Based on varying findings of the potency of PARP inhibition on plaque stability we investigated the effect of PARP inhibition on plaque stability. We are in line with the finding of reduced apoptosis in the lesion and the possible relevance of caspase $3[7,9]$, which may represent a possible mechanism of PARP inhibition on atherosclerotic lesion development. But by INO-1001 we were not able to ascertain an effect on an increase in collagen content or amount of VSMCs in the lesion of the therapy group. In the current experimental setting with the inhibitor INO-1001 of the present study, PARP inhibition reduces cell apoptosis in the lesion, but does not seem to increase plaque stability.

Evidence from human and experimental studies suggests a dominant role of the $\mathrm{T}$ cell immune response, especially Th1 cells, in atherogenesis [13]. A previous study of our group shows that PARP inhibition with INO-1001 suppresses both alloantigen-dependent (MLR) and antigen-independent (PHA) T-cell proliferation [14]. In addition, previously it was shown that $\mathrm{T}$ cells are reduced in PARP inhibited mice[10]. By investigating the lesional $\mathrm{T}$ cell infiltration content the present study demonstrates a reduced $\mathrm{T}$ cell number in the lesion of INO-1001 treated mice, which is in line with previous result. Due to the inhibitory effect of PARP inhibition on $\mathrm{T}$ cell recruitment and proliferation, the study investigated the impact of PARP inhibition on $\mathrm{T}$ cell activation. The present study shows that blockade of PARP results in reduced auto-antigen oxLDL dependent activation of peripheral lymphocytes. Several cytokines such as If $n \gamma$ and Il 2 were downregulated by the PARP inhibitor INO1001. Thus, the effects of INO-1001 on T cell proliferation, activation and lesional invasion may represent a possible mechanism in atherogenesis.

It is known that PARP-1 inhibition influences the expression of adhesion molecules. Matter et al. and others clearly showed an inhibitory effect of PARP inhibition on the expression of adhesion molecules $[9,16]$. The current study is in line with the finding of reduced numbers of the adhesion molecule VCAM-1, an important adhesion molecule and a marker of cellular activation, in atherosclerotic lesions. In addition, due to these results the study focused on the VCAM-1 expression on endothelial cells, which are known to be involved in initiation and progression of atherogenesis. We observed a lower number of VCAM- $1^{+}$endothelial cells (EC) in INO-1001 treated mice. Thus, the effect of PARP inhibition on adhesion molecule, especially on endothelial cells and thus on the recruitment of inflammatory cells ( $\mathrm{T}$ cells, dendritic cells) in atherogenesis might be a potent mechanism.

A previous study of our group showed that activated and fully mature DCs, identified by surface $C d 83$, are present in the inflammatory infiltrate characteristic for unstable carotid and coronary atheroma [3]. Such DCs produce chemokines and thus can regulate cell transmigration into the lesion [3]. An influence of PARP in the maturation of DC has been already described in vitro, but not in vivo [6]. The present study demonstrates reduced numbers of DCs in the adventitia, located next to atherosclerotic lesions, but not in the plaque of INO-1001 treated compared to control mice. The study further showed reduced levels of the chemokine MIP-3 $\alpha$ (CCL20), which is commonly upregulated after inflammatory or 'T cell signals, in INO1001 treated animals compared to controls. MIP-3 $\alpha$ binds to CCR6, primarily on immature DC, in order to recruit them to the site of inflammation. DCs in addition seem to be less activated in INO-1001 treated compared to control mice. Thus, the reduced presence and grade of activation of DC, a reduced transmigration as well as the loss of chemoattractant for immature DC may provide a possible mechanism of PARP inhibition in the development of atherosclerosis.

Modified lipids such as oxidized LDL (oxLDL), in part presented by mature dendritic cells and $M \varnothing$, give rise to clonal expansion of oxLDL-specific T cells and generation of autoantibodies specific for these oxLDL compounds $[18,19]$. The presence of autoantibodies against oxLDI are considered biological indices of LDL in vivo oxidation. Interestingly, divergent findings between IgM- and IgG-anti-oxLDL autoantibodies in cardiovascular disease are being increasingly reported [20-22]. Our study shows, that IgM, but not IgG autoantibody serum levels to oxLDL were significantly reduced in INO-1001 treated mice. Thus, in the experimental setting of the present study IgM (auto)antibody levels to oxLDL may have atheroprotective effects.

INO-1001 treated mice exhibited normal fertility, development and growth. Plasma cholesterol and triglycerides were equivalent between both groups. Previous studies, investigating the role of inflammatory cytokines such as IL-17A[11] and IL-18 [23] in Apoe $\%$ mice also find no effects of these pro-inflammatory cytokines on plasma cholesterol levels. Here we report that INO-1001 treated mice have reduced atherosclerotic lesions in the presence of unchanged serum cholesterol and HDL/LDL ratio. These findings suggest that PARP is unlikely to affect atherogenesis via changes in hyperlipidemia as it was shown to have an impact on atherosclerosis [25].

In conclusion, the study is in line with the previous finding that pharmacologic PARP inhibition, this time by INO-1001, attenuates atherogenesis. The study not only underlines the potential inhibitory effect on the inflammatory compound in atherosclerotic lesion, but also helps to provide further information for a better understanding of already known mechanisms. Even more important, the current study shows new findings providing possible additional mechanisms including the effects of PARP inhibition on atherogenic cells (DC, T cells and EC) as well as on IgM auto-antibody serum levels to oxLDL. The differences in the results of some mechanisms may be based on the type and dosage of PARP inhibitor used in the study and needs to be further elaborated. However, based on these findings, PARP action appears to represent a crucial pathway in the pathogenesis of atherosclerosis, potentially amenable to novel treatment options. 


\section{REFERENCES}

1. Ross R. Atherosclerosis--an inflammatory disease. N Engl J Med. 1999; 340:115-26.

2. Hansson GK, Libby P. The immune response in atherosclerosis: a double-edged sword. Nat Rev Immunol. 2006; 6:508-19.

3. Erbel C, Sato K, Meyer FB, Kopecky SI, Frye RL, Goronzy JJ, Weyand CM. Functional profile of activated dendritic cells in unstable atherosclerotic plaque. Basic Res Cardiol. 2007; 102:123-32.

4. Szabo C, Zingarelli B, O'Connor M, Salzman AL. DNA strand breakage, activation of poly (ADP-ribose) synthetase, and cellular energy depletion are involved in the cytotoxicity of macrophages and smooth muscle cells exposed to peroxynitrite. Proc Natl Acad Sci U S A. 1996; 93:1753-8

5. Pacher P, Szabo C. Role of the peroxynitrite-poly(ADPribose) polymerase pathway in human disease. Am J Pathol. 2008; 173:2-13.

6. Aldinucci A, Gerlini G, Fossati S et al. A key role for poly(ADP-ribose) polymerase-1 activity during human dendritic cell maturation. J Immunol. 2007; 179:305-12.

7. Hans CP, Zerfaoui M, Naura AS, Catling A, Boulares $\mathrm{AH}$. Differential effects of PARP inhibition on vascular cell survival and ACAT-1 expression favouring atherosclerotic plaque stability. Cardiovasc Res. 2008; 78:42939.

8. Oumouna-Benachour K, Hans CP, Suzuki Y, et al. Poly(ADP-ribose) polymerase inhibition reduces atherosclerotic plaque size and promotes factors of plaque stability in apolipoprotein E-deficient mice: effects on macrophage recruitment, nuclear factor-kappaB nuclear translocation, and foam cell death. Circulation. 2007; 115:2442-50.

9. Xie JJ, Yu X, Liao YH et al. Poly (ADP-Ribose) polymerase inhibition attenuates atherosclerotic plaque development in ApoE $/-$ mice with hyperhomocysteinemia. J Atheroscler Thromb. 2009; 16:641-53.

10. von Lukowicz T, Hassa PO, Lohmann C et al. PARP1 is required for adhesion molecule expression in atherogenesis. Cardiovasc Res. 2008; 78:158-66.

11. Erbel C, Chen L, Bea F et al. Inhibition of II-17A attenuates atherosclerotic lesion development in ApoE-deficient mice. J Immunol. 2009; 183:8167-75.

12. Nakashima Y, Plump AS, Raines EW, Breslow JL, Ross R. ApoE-deficient mice develop lesions of all phases of atherosclerosis throughout the arterial tree. Arterioscler Thromb. 1994; 14:133-40.

13. Zhou X, Paulsson G, Stemme S, Hansson GK. Hypercholesterolemia is associated with a T helper (Th) $1 / \mathrm{Th} 2$ switch of the autoimmune response in atherosclerotic apo E-knockout mice. J Clin Invest. 1998; 101:1717-25.

14. Szabo G, Bahrle S, Sivanandam V et al. Immunomodulatory effects of poly(ADP-ribose) polymerase inhibition contribute to improved cardiac function and survival during acute cardiac rejection. J Heart Lung Transplant. 2006; 25:794-804.
15. Davenport $P$, Tipping $P G$. The role of interleukin-4 and interleukin-12 in the progression of atherosclerosis in apolipoprotein E-deficient mice. Am J Pathol. 2003; 163:1117-25.

16. Hans CP, Zerfaoui M, Naura $A S$ et al. Thienol2,3-c]isoquinolin-5-one, a potent poly(ADP-ribose) polymerase inhibitor, promotes atherosclerotic plaque regression in high-fat diet-fed apolipoprotein E-deficient mice: effects on inflammatory markers and lipid content. J Pharmacol Exp Ther. 2009; 329:150-8.

17. Radovits T, Lin LN, Zotkina J et al. Poly(ADP-ribose) polymerase inhibition improves endothelial dysfunction induced by reactive oxidant hydrogen peroxide in vitro. Eur J Pharmacol. 2007; 564:158-66.

18. Palinski W, Witztum JL. Immune responses to oxidative neopitopes on IDL and phospholipids modulate the development of atherosclerosis. J Intern Med. 2000; 247:371-80.

19. Palinski W, Rosenfeld ME, Yla-Herttuala S et al. Low density lipoprotein undergoes oxidative modification in vivo. Proc Natl Acad Sci U S A. 1989; 86:1372-6.

20. Tsimikas S, Bergmark C, Beyer RW et al. Temporal increases in plasma markers of oxidized low-density lipoprotein strongly reflect the presence of acute coronary syndromes. J Am Coll Cardiol. 2003; 41:360-70.

21. Dotevall A, Hulthe J, Rosengren A, Wiklund O, Wilhelmsen L. Autoantibodies against oxidized low-density lipoprotein and $\mathrm{C}$-reactive protein are associated with diabetes and myocardial infarction in women. Clin Sci (Lond). 2001; 101:523-31.

22. Wu R, de Faire U, Lemne C, Witztum JL, Frostegard J. Autoantibodies to OxLDL are decreased in individuals with borderline hypertension. Hypertension. 1999; 33:539.

23. Whitman SC, Ravisankar P, Daugherty A. Interleukin-18 enhances atherosclerosis in apolipoprotcin $\mathrm{E}(-/)$ mice through release of interferon-gamma. Circ Res. 2002; 90:E34-8.

24. Coats AJ. Ethical authorship and publishing. Int J Cardiol. 2009; 131:149-50.

25. Evans D, Bode A, von der Lippe G, Beil FU, Mann WA. Cerebrovascular atherosclerosis in type III hyperlipidemia is modulated by variation in the apolipoprotein A5 gene. Eur J Med Res. 2011; 16(2):79-84.

Received: November 25, 2010 / Accepted: April 18, 2011

\section{Address for correspondence:}

Dr. med. Christian Erbel

Department of Cardiology, Angiology and Pneumology

Medical Clinic III

University Hospital Heidelberg

INF 410

69120 Heidelberg

Germany

Phone: +49-6221-56-38879

Fax: +49-6221-56-5515

E-mail: Christian.Erbel@med.uni-heidelberg.de 